\title{
Solasonine Induces Apoptosis and Inhibits Proliferation of Bladder Cancer Cells by Suppressing NRP1 Expression
}

\author{
Yang Dong, ${ }^{1,2,3}$ Lin Hao, ${ }^{1,2,3}$ Zhen-duo Shi, ${ }^{1,2}$ Kun Fang, ${ }^{4}$ Hui Yu, ${ }^{5}$ Guang-hui Zang, \\ Tao Fan, ${ }^{1,2}$ and Cong-hui Han $\mathbb{D}^{1,2,3}$ \\ ${ }^{1}$ Department of Urology, Xuzhou Central Hospital, Xuzhou, Jiangsu Province, China \\ ${ }^{2}$ Xuzhou Clinical School of Xuzhou Medical University, Xuzhou, Jiangsu Province, China \\ ${ }^{3}$ Medical College of Soochow University, Soochow, Jiangsu Province, China \\ ${ }^{4}$ Department of Nephrology, The Third Affiliated Hospital of Shandong First Medical University, Jinan, \\ Shandong Province, China \\ ${ }^{5}$ Department of Urology, Yantai Hospital of Traditional Chinese Medicine, Yantai, Shandong Province, China
}

Correspondence should be addressed to Cong-hui Han; hanchdoctor@st.btbu.edu.cn

Received 31 January 2022; Accepted 11 February 2022; Published 2 March 2022

Academic Editor: Dong-Hua Yang

Copyright $(2022$ Yang Dong et al. This is an open access article distributed under the Creative Commons Attribution License, which permits unrestricted use, distribution, and reproduction in any medium, provided the original work is properly cited.

\begin{abstract}
Solasonine, a steroidal alkaloid extracted from Solanum nigrum L., has been found to exert inhibitory effect on cancers. However, the underlying anticancer mechanisms of solasonine, particularly in urinary bladder cancer (BC), remain unclear. In this study, we identified the potential targets and biological functions associated with solasonine activity using a bioinformatics approach. Ingenuity pathway analysis revealed that neuropilin-1 (NRP1) and other signaling pathways, such as PI3K/AKT and ERK/MAPK pathways, were potentially involved in the therapeutic effects of solasonine. The ability of solasonine in inducing apoptosis and inhibiting proliferation in BC cells was confirmed experimentally, and the inhibition of ERK/MAPK, P38/MAPK, and PI3K/AKT pathways was validated by Western blot. Mechanistically, solasonine suppressed the expression of NRP1 protein, but not that of mRNA. Further results of molecular docking and molecular dynamics simulation analysis indicated that solasonine could directly bind to the b1 domain of NRP1 protein with a reasonable and stable docking conformation. We previously found that targeting NRP1 is a potential antitumor strategy. Combined with these findings, it can be speculated that the binding of solasonine with NRP1 on the cell membrane could prevent the formation of NRP1/VEGFA/VEGFR2 and NRP1/EGFR complexes, resulting in the inhibition of downstream signaling, including ERK/MAPK, P38/MAPK, and PI3K/AKT pathways. Additionally, intracellular solasonine could inhibit the membrane localization of NRP1 and provoke its cytoplasmic retention, facilitating the degradation of NRP1 protein in the cytoplasm. The dual effects induced by the binding of solasonine to NRP1 extracellularly and intracellularly could account for the antiproliferative and proapoptotic effects of solasonine on BC.
\end{abstract}

\section{Introduction}

Urinary bladder cancer (BC), one of the leading causes of cancer-associated death, is the tenth most common malignancy worldwide. According to the Global Cancer Statistics, approximately 570,000 new cases and 210,000 BC-related deaths were recorded during 2020 [1]; and these values increase annually, with approximately 81,180 new cases and 17,100 deaths expected to occur in the United States in 2022 [2]. Urothelial carcinoma is the most prevalent histological phenotype and accounts for approximately $90 \%$ of BC cases. Due to its high recurrence rate and imperceptible symptoms, the long-term outcomes and quality of life of BC patients remain poor. Postoperative recurrence has been reported in approximately $70 \%$ of patients, and approximately $20 \%$ of these patients may progress to muscle invasion with distant metastatic potential [3-5]. Metastatic BC has a poor prognosis with a 5 -year survival rate of only $5 \%[5,6]$. Moreover, based on tumor stage and grade, long-term follow-up endoscopic examinations and treatment with intravesical 
Bacillus Calmette-Guérin instillation are required, together with cisplatin-based combination chemotherapy and radiotherapy. Consequently, $\mathrm{BC}$ is associated with high financial and psychological burdens.

Emerging immunotherapeutic approaches such as checkpoint inhibitors and tumor-targeted CAR-T cells have shown efficacy in improving the prognosis of patients with advanced BC, but only a fraction of patients can achieve durable clinical responses [6-9]. Moreover, high costs restrict the clinical application of these approaches. Therefore, exploring novel, affordable, and efficacious anticancer drugs with low toxicity is of great clinical importance. Currently, molecular-targeted therapy against cancer has attracted attention worldwide, presenting novel frontiers in $\mathrm{BC}$ treatment. Molecular targeting agents such as those targeting vascular endothelial growth factor (VEGF), epidermal growth factor receptor (EGFR), fibroblast growth factor receptor, and mammalian target of rapamycin have been successfully used in several types of cancers, presenting encouraging results in extending patient survival $[6,7,10]$. In addition, certain factors have been identified in the occurrence and progression of BC, including Erb-B2 receptor tyrosine kinase 2, avian myelocytomatosis viral oncogene homolog (MYC), cyclin D1, and hypoxia-inducible factor $1 \alpha$ $(\mathrm{HIF}-1 \alpha)$, that are important for clinical applications $[6,10]$. Cancer development is a multistage process involving aberrant cell proliferation, apoptosis, invasion, metastasis, and metabolism that are typically linked to the deregulation of tumor-related genes [11]. These regulatory factors are candidate targets associated with improved treatment efficacy for BC.

Natural plants are an important source of anticancer agents. Solanum nigrum L. (S. nigrum) is an annual herbaceous plant that has been used as a folklore medicine in China for BC therapy since ancient times [12, 13]. In a previous study, we explored the potential mechanisms underlying $S$. nigrum activity against BC. It was found that it exerted its therapeutic effects via multiple signaling pathways, including the MAPK, PI3K/AKT, and HIF-1 pathways [13]. S. nigrum contains two main kinds of effective components, namely, solanine and Solanum nigrum polysaccharide [14]. Solasonine is the major contributor to glycoalkaloid solanine, which can be extracted from all parts of the plant and unripe fruits $[14,15]$. Since it was first identified in Solanum by Oddo and Cesaris in 1911, solasonine has been isolated from over 100 species and has shown beneficial pharmacological effects, such as anticancer and antimicrobial activities [14, 16]. Accumulating studies have suggested that solasonine has multiple antitumor activities, including suppression of proliferation, promotion of apoptosis, and inhibition of metastasis in a variety of tumor cells [17-19]. Solasonine could suppress glioma growth by inhibiting proinflammatory mediators and the activation of the P38/MAPK and JNK/MAPK pathways [20]. The activation of AMPK/FOXO3a axis has also been reported to be involved in the inhibition of cell proliferation mediated by solasonine in acute monocytic leukemia. [17]. In addition, another study showed that solasonine is a potential anticancer agent candidate for hepatocellular carcinoma, which can mediate the reciprocal regulation of miR-375-3p and lncRNA CCAT1, resulting in the downregulation of interferon regulatory factor 5 [18]. However, the effects of solasonine on BC have not been studied, and the underlying anticancer mechanism remains to be elucidated.

Neuropilin-1 (NRP1) is a novel target and immunomodulator for cancer therapy [21-24]. Our previous study demonstrated that NRP1 was overexpressed in BC, while downregulation of NRP1 promoted BC cell apoptosis and inhibited BC cell proliferation, invasion, and migration [24]. In the present study, we identified the potential targets and pathways associated with the activity of solasonine against BC using a bioinformatics approach. We found that solasonine may interact with NRP1 protein. We further explored the potential mechanism by which solasonine influences tumor biology in BC and its effect on NRP1 expression in vitro. Our results demonstrated that solasonine significantly inhibited BC cell proliferation and promoted cell apoptosis by inhibiting the MAPK and PI3K/ AKT signaling pathways. NRP1 may be a potential effector of solasonine in mediating anti-BC responses.

\section{Materials and Methods}

2.1. The Molecular Structure of Solasonine. The molecular structure of solasonine was generated using the JSME platform (https://jsme-editor.github.io/) [25] by importing the SMILES code obtained from the PubChem database (https://pubchem.ncbi.nlm.nih.gov/). The molecular structure was converted to a $3 \mathrm{D}$ image using the Yinfo Cloud platform (http://cloud.yinfotek.com/).

2.2. Evaluation of Pharmacokinetics Properties for Solasonine. The Traditional Chinese Medicines for Systems Pharmacology (TCMSP) database and analysis platform server (https://tcmsp-e.com/) is a systems-level pharmacology database, which can calculate pharmacokinetics properties for naturally occurring compounds based on ADME (absorption, distribution, metabolism, and excretion) principle. The TCMSP server was used to evaluate the pharmacokinetics characteristics of solasonine on ADME-related properties, such as human oral bioavailability (OB), druglikeness (DL), Caco-2 permeability (Caco-2), blood-brain barrier (BBB) permeability, and Lipinski's rule of five (MW, AlogP, TPSA, Hdon, Hacc) [26].

2.3. Potential Targets Prediction for Solasonine. Potential targets of solasonine were predicted using an integrative application of PharmMapper (http://www.lilab-ecust.cn/ pharmmapper/; updated on January 01, 2016) [27, 28], TargetNet (http://targetnet.scbdd.com/, updated on February 25,2014$)$ [29], and similarity ensemble approach (SEA, http://sea.bkslab.org/, updated on March 26, 2019) servers [30]. These databases adopted multiple prediction algorithms to generate compound target genes. All genes were converted to the HUGO Gene Nomenclature Committee (HGNC, https://www.genenames.org/) symbols to avoid confusion across platforms. 
2.4. Bioinformatics Analysis by Ingenuity Pathway Analysis (IPA). The potential target genes of solasonine were analyzed by IPA, which can predict downstream effects and identify new targets or candidate biomarkers and can obtain data analysis and interpretation to understand the experimental results within the context of biological systems [24]. In this study, analyses in five modules were performed using IPA, including canonical pathway analysis, disease and function analysis, upstream analysis, causal regulatory network analysis, and toxicity analysis.

2.5. Identification of the Targets of $B C$. The genes associated with $\mathrm{BC}$ were collected from five sources, including the databases of GeneCards (https://www.genecards.org/; version 5.5) [31], Online Mendelian Inheritance in Man (OMIM) (http://www.omim.org/; updated on September 03, 2021) [32], Therapeutic Target Database (TTD, http://db. idrblab.net/ttd/; updated on June 01, 2020) [33], Pharmacogenomics Knowledge Base (PharmGKB, https://www. pharmgkb.org/) [34], and DrugBank (https://www. drugbank.com/; version 5.1.8) [35]. We searched these databases with "bladder cancer," "bladder carcinoma," and "bladder tumor" as the keywords.

2.6. GO and KEGG Enrichment Analyses. As described in our previous research [24], GO analysis is a commonly used method to annotate genes and gene products for their molecular functions and associated biological pathways and cellular components [36]; KEGG is a useful resource for the systematic analysis of gene functions and related high-level genome functional information $[37,38]$. In this study, the DOSE [39] and clusterProfiler [40] packages of the statistical software R (version 4.1.0) were used for mining information related to the biological effects of differential expressed genes and implementing pathway enrichment analysis. Subsequently, the enrichplot and ggplot2 packages were used for high-quality graph generation.

2.7. Network Analysis. The gene-gene interaction network was generated using GeneMANIA prediction server (http:// www.genemania.org/) [41], which can also reveal the biological functions of the gene set. After KEGG pathway and GO terms analyses, the representative enriched KEGG and GO terms and their related gene symbols were imported into Cytoscape software (version 3.8.2) [42] to construct the gene-pathway/GO term network.

2.8. Molecular Docking Analysis. Molecular docking analysis was conducted to evaluate the binding affinity of solasonine towards NRP1 protein. The 3D structure of solasonine was optimized with energy minimization in the MMFF94 force field. The crystal structure of human NRP1 (PDB ID: 1KEX, NRP1-b1 domain) was directly retrieved from the RCSB protein data bank (PDB) database (https:// www.rcsb.org/). The protein structures were then prepared by adding hydrogen atoms and merging nonpolar hydrogen atoms using AutoDock Tools (ADT, version 1.5.6)
[43] and saved as PDBQT files. The docking pocket of protein was defined using the grid box module. AutoDock Vina (version 1.1.2) [44] was utilized to perform molecular docking, and after evaluating the binding energy (affinity), the conformation with the best affinity was visualized in PyMOL (version 2.5.2) software. The $2 \mathrm{D}$ diagram of the protein-ligand was visualized using the Discovery Studio 2019 server.

2.9. Molecular Dynamics Simulation and Binding Free Energy Calculation. The protein-ligand docked complex structure of NRP1-solasonine was prepared for molecular dynamics simulation (MDS) using Visual Molecular Dynamics (VMD, version 1.9.4) software [45]. The TIP3P water model was adopted to solvate the complexes followed by adding ions to neutralize. Periodic boundary conditions were employed. Energy minimization was performed with a tolerance of $1000 \mathrm{~kJ} / \mathrm{mol} / \mathrm{nm}$, and the system was equilibrated by NVT (constant volume) of $1 \mathrm{~atm}$ and NPT (constant pressure) of $300 \mathrm{~K}$ ensemble for 100 picoseconds (ps). Electrostatic interaction calculations and constraint on bond length were performed using the particle mesh Ewald (PME) method and the linear constraint solver (LINCS) algorithm, respectively. MDS was carried out applying the OPLS force field for 200 nanoseconds (ns) by GROMACS (version 2021.3) [46], with trajectories generated every 2 femtoseconds (fs) and collected every 2 ps. Several parameters including root-mean-square deviation (RMSD), root-mean-square fluctuation (RMSF), radius of gyration (Rg), and number of hydrogen bonds were measured to assess the conformational and performance stability of the protein-ligand complex in a dynamic environment. Finally, the binding free energy of the docked complex and the energy contribution of each residue to binding were investigated using the molecular mechanics Poisson-Boltzmann surface area (MM-PBSA) method [47] implemented in the AmberTools21 package.

2.10. Cell Culture and Reagents. The human bladder cancer cell lines T24 and 5637 were purchased from the Cell Resource Center of the Shanghai Institutes for Biological Sciences, Chinese Academy of Sciences (Shanghai, China). Solasonine was purchased from Chengdu Must Bio-Technology Company (Chengdu, Sichuan, China). The cell lines were cultured in RPMI-1640 medium with $100 \mathrm{U} / \mathrm{mL}$ penicillin, $100 \mu \mathrm{g} / \mathrm{mL}$ streptomycin, and $10 \%$ fetal bovine serum at $5 \% \mathrm{CO}_{2}$ in a $37^{\circ} \mathrm{C}$ humidified culture environment, which was as previously described [24, 48]. Short-tandem repeat profiling was used to authenticate the cell lines less than 6 months before this project was initiated, and the cells were not in culture for more than 2 months.

2.11. Cell Viability Assay. T24 and 5637 cells were seeded in 96-well cell culture plates at an initial density of $0.2 \times 10^{4}$ cells/well in quintuplicate and treated with increasing concentrations of solasonine or vehicle (DMSO) for $48 \mathrm{~h}$. 
The cell counting kit-8 (CCK8) assay was used to measure cell viability. Lastly, optical density (OD) was measured at $450 \mathrm{~nm}$ to detect viable cells.

2.12. CCK8 Assay. T24 and 5637 cells were seeded in 96-well cell culture plates at an initial density of $0.2 \times 10^{4}$ cells/well in triplicate. After treating with solasonine or vehicle (DMSO) for $24 \mathrm{~h}$, the medium was discarded and the cells were cultured in a resuspended RPMI-1640 medium at a volume of $100 \mu \mathrm{L} /$ well. At different time points, the cells were incubated with $10 \mu \mathrm{L}$ CCK8 reagent (CK04, Dujindo Chemical, Japan) at $37^{\circ} \mathrm{C}$ for $4 \mathrm{~h}$. Finally, the OD of each well at $450 \mathrm{~nm}$ wavelength was measured to evaluate cell proliferation.

2.13. Flow Cytometric Apoptosis Test. As performed in our previous study [24], T24 and 5637 cells were digested with $0.25 \%$ trypsin, washed with PBS, and centrifuged at $1300 \mathrm{rpm}$ for $3 \mathrm{~min}$. The supernatant was aspirated followed by adding $200 \mu \mathrm{L}$ of $1 \times$ binding buffer to each tube. Next, $5 \mu \mathrm{L}$ of Annexin-V-APC (eBioscience, $88-8007$ ) and $5 \mu \mathrm{L}$ of propidium iodide (PI) (Sigma) were added to each tube. The tubes were then incubated for $15 \mathrm{~min}$ at room temperature and shielded from light. Then, a BD FACSCalibur flow cytometer (BD Biosciences) was used to assess the cell apoptosis.

2.14. Flow Cytometry Cell Cycle Analysis. As described previously [24], cells were digested with $0.25 \%$ trypsin, washed with PBS, and centrifuged at $1300 \mathrm{rpm}$ for $3 \mathrm{~min}$. The cell pellet was washed twice with PBS and resuspended in $1 \mathrm{~mL}$ of PBS. Next, the tubes were oscillated on a low-speed oscillator, and $3 \mathrm{~mL}$ of $70 \%$ ice-cold ethanol was added to fix the cells for $2 \mathrm{~h}$ at $4^{\circ} \mathrm{C}$. After discarding ethanol, the pellet was resuspended in $2 \mathrm{~mL}$ PBS and centrifuged at $3000 \mathrm{rpm}$ for $3 \mathrm{~min}$ followed by removing the supernatant. Subsequently, $1 \mathrm{~mL}$ DNA staining solution (CCS102, MultiSciences, China) and $10 \mu \mathrm{L}$ permeabilization solution (CCS102, MultiSciences) were treated and the tubes were incubated at room temperature for $30 \mathrm{~min}$ in the dark. Finally, cell cycle detection was performed using a NovoCyte 2060R flow cytometer (ACEA Biosciences, USA) and analyzed by FlowJo software (Tree Star, Inc, USA).

2.15. Protein Extraction and Western Blot. Total protein was extracted from T24 and 5637 cells, and Western blot were performed as described previously [24, 49]. Briefly, equal protein amounts $(20 \mu \mathrm{g})$ were loaded into each lane. The primary antibodies for Western blot are listed below: NRP1 (1:500; ab25998, Abcam, USA), extracellular signal-regulated protein kinases 1 and 2 (ERK1/2) $(1: 3000$; \#9107, Cell Signaling Technology Co., Ltd. (CST), China), phosphorylated ERK (p-ERK) (1:500; \#4376, CST), p38 mitogenactivated protein kinase (P38) (1:3000; \#8690, CST), phosphorylated P38 (p-P38) (1 : 500; \#4631, CST), mitogenactivated protein kinase kinase 1 (MEK1) (1:3000; ab32091, Abcam), phosphorylated MEK 1 and 2 (p-MEK1/ 2) $(1: 1000 ; \# 9154, \mathrm{CST})$, PI 3 kinase (PI3K) (1:1000; AF1729, Beyotime, China), phosphorylated PI3K (p-PI3K) (AF3242, Affinity), threonine-protein kinase (AKT) (1: 1000; AA326, Beyotime), phosphorylated AKT (p-AKT) (1:200; ab81823, Abcam), EGFR (1:500; GB11084, Servicebio, China), vascular endothelial growth factor A (VEGFA) (1:500; GB11034B, Servicebio), glyceraldehyde3-phosphate dehydrogenase (GAPDH) $(1: 1000 ; \mathrm{MB} 001 \mathrm{H}$, BioWorld, USA), and $\beta$-actin $(1: 10000$; TDY051, Beijing TDY Biotech Co., Ltd., China). After washing with TBST, the membranes were further incubated for $2 \mathrm{~h}$ with a secondary anti-mouse ( $1: 10000$; AS1106, Aspen Biotechnology Co., Ltd. (ASPEN), China) or anti-rabbit (1:10000; AS1107, ASPEN) antibody, as appropriate. The presentation of target protein bands was enhanced using chemiluminescence (Millipore) and then quantified by densitometry (BioRad image analysis system) and normalized to $\beta$-actin levels.

2.16. Statistical Analysis. Statistical analyses were conducted using SAS 9.43 statistical software (SAS Institute Inc., Cary, NC, USA). One-way ANOVA was carried out to perform significance tests for multiple groups. Significant differences for two groups were evaluated using Student's t-test. A $P<0.05$ was considered statistically significant.

\section{Results}

3.1. Pharmacokinetics Properties and Potential Targets of Solasonine. The molecular structure of solasonine is shown in Figure 1(a), and its ADME-related pharmacokinetic properties are presented in Table 1. Notably, the OB of solasonine was $25.94 \%$. Subsequently, the potential targets of solasonine were predicted using three different approaches described in Methods. Figure 1(b) shows 286 candidate targets identified from PharmMapper (Norm fit $>0.3$ ), five targets from the TargetNet server, and 16 human target proteins from the SEA database. Three genes, AR, G6PD, and CYP17A1, shared by the two databases, and a total of 304 unique genes in the merged union were identified as potential targets of solasonine for further analysis. Information on potential target genes is provided in Table S1.

3.2. Bioinformatics Analysis by IPA. The canonical pathway analysis generated by IPA revealed that the potential target genes of solasonine were significantly enriched in 494 canonical pathways, including Hippo signaling $(-\log \mathrm{P}=6.66)$, cell cycle $(-\log \mathrm{P}=6.44), \mathrm{HIF}-1$ signaling $(-\log \mathrm{P}=3.28)$, $\mathrm{PI} 3 \mathrm{~K} / \mathrm{AKT}$ signaling $(-\log \mathrm{P}=1.72)$, and ERK/MAPK signaling $(-\log \mathrm{P}=3.13)$ (Figure $1(\mathrm{c})$ and Table S2). The disease and functional analysis showed 500 remarkably enriched annotations of diseases or functions that were most related to cell death and survival, cell cycle, and various cancer processes (Figure $1(\mathrm{~d})$ and Table S3). Upstream regulatory and causal regulatory network analyses were performed to predict the potential upstream regulators of solasoninetargeting genes (Table S4) and the regulators of these 

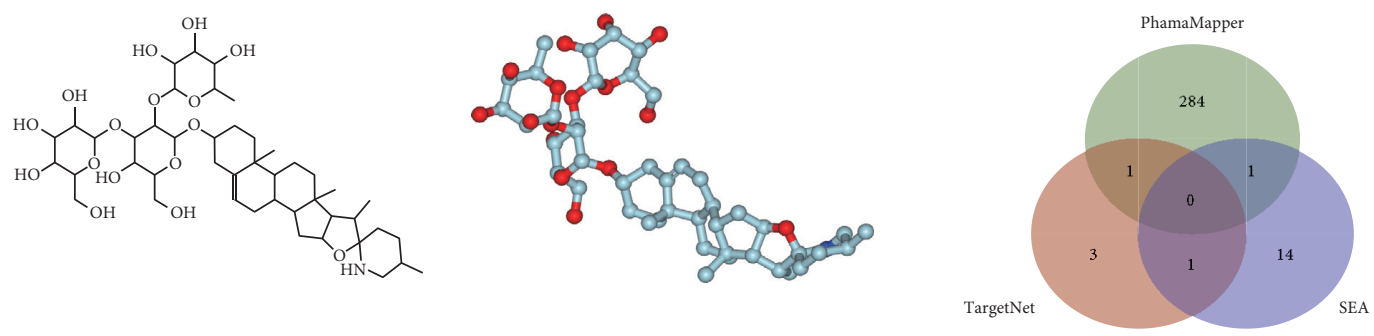

(a)

(b)

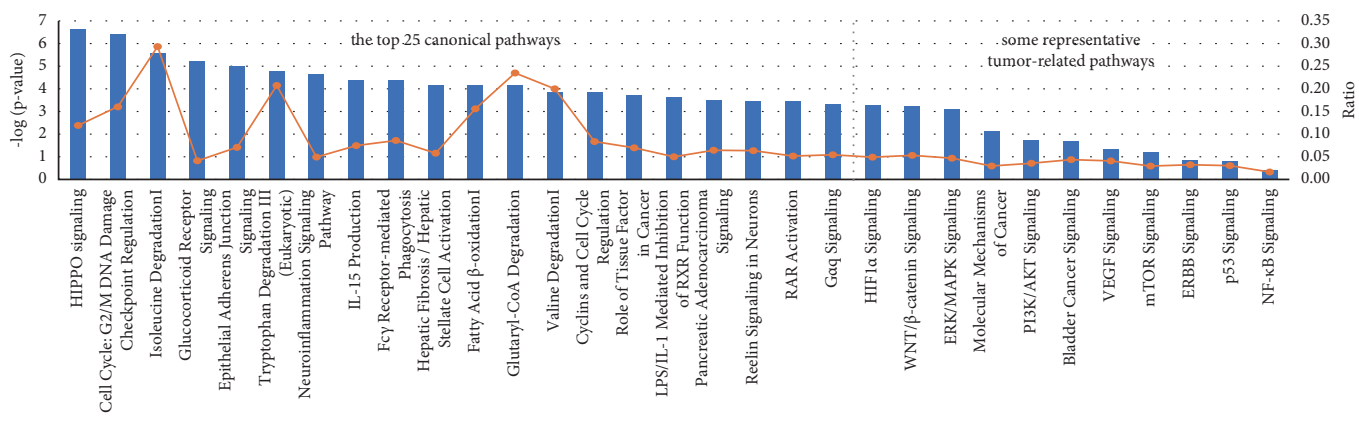

- - log (p-value)

(c)
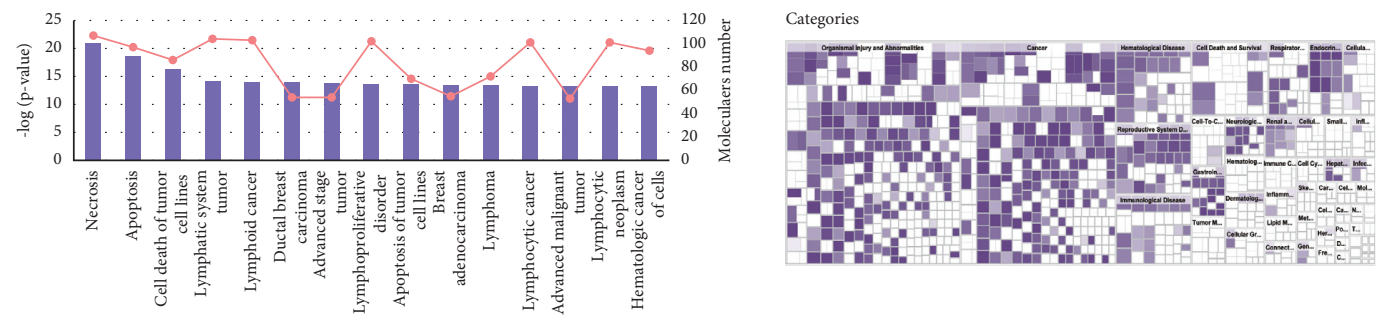

-log (p-value)

(d)
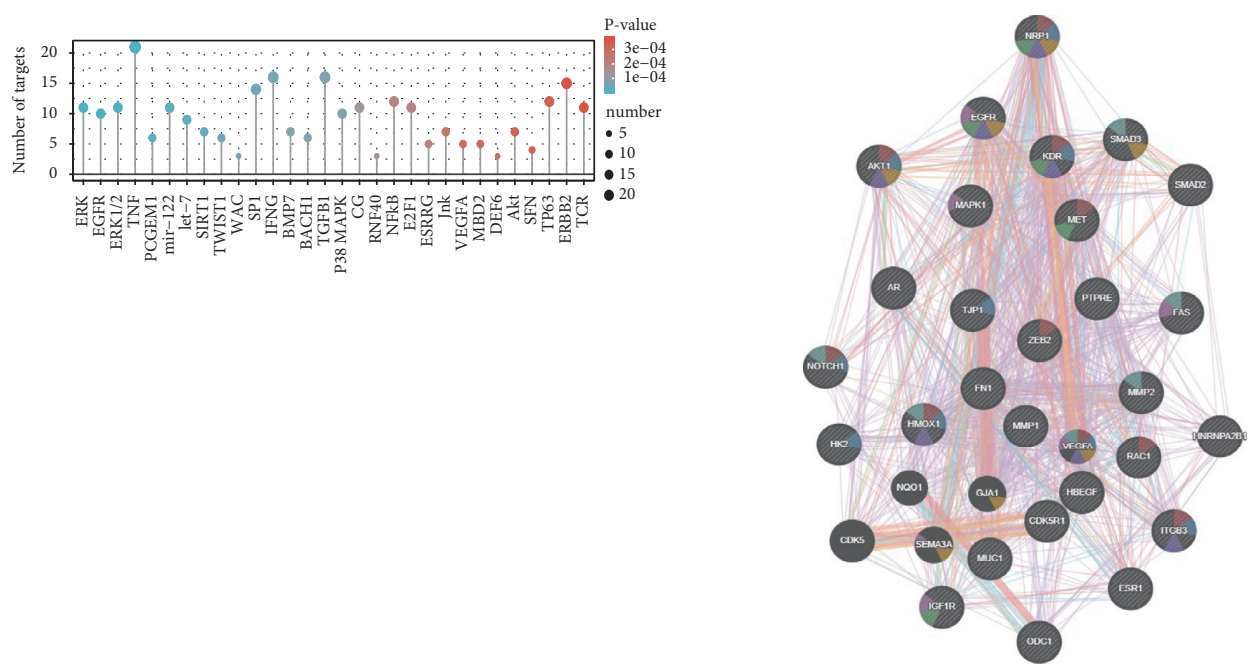

Networks

Physical Interactions (70.90\%) Co-expression (16.01\%)

Predicted (4.96\%)

Functions

tissue migration

Co-localization $(3.22 \%$

angiogenesis

Genetic Interactions (2.63\%)

Shared protein domains $(0.55 \%)$

regulation of cell growth

- epithelial cell proliferation

protein tyrosine kinase activity
stress-activated MAPK cascade

(f)

FIgure 1: Continued. 


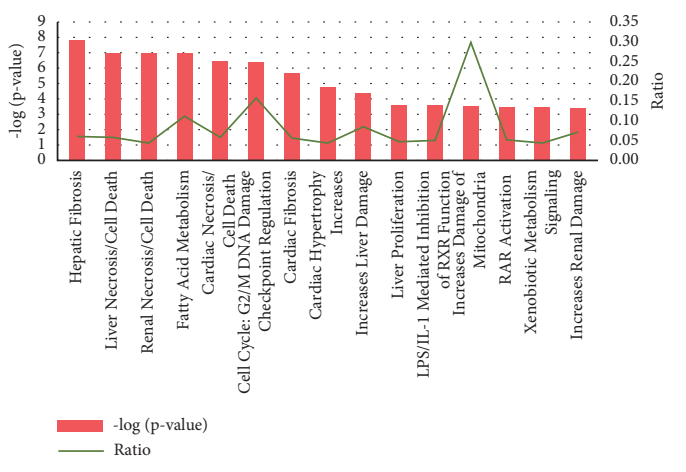

(g)

FIGURE 1: Bioinformatics analysis of potential targets of solasonine using IPA. (a) 2D and 3D molecular structures of solasonine. (b) Venn graph showing overlapping and specific genes predicted from PharmMapper, TargetNet, and SEA databases. (c) Canonical pathway analysis of solasonine by IPA. The top 25 enriched canonical pathways and some representative tumor-related pathways, sorted by - $\log (P$-value $)$ in decreasing order, are shown. (d) Disease and functional analysis using IPA for solasonine. The bar graph shows the top 15 annotations, and the heatmap shows the enrichment of targeting genes enriched in disease and function categories. (e) Upstream analysis using IPA for solasonine, showing the upstream regulatory factors for potential target genes of solasonine. The top 30 upstream regulatory factors in decreasing order of $P$-value were shown. (f) Causal regulatory network associated with NRP1. NRP1 was predicted as a master regulator involved in the regulation of 7 upstream regulators, including ERK (group), EGFR, AKT, KDR, MET, SMAD2, SMAD3. (g) Toxicity analysis for solasonine shows the top 15 potential toxicities.

TABle 1: Pharmacological and molecular properties of solasonine.

\begin{tabular}{lccccccccccc}
\hline Name & MW & AlogP & Hdon & Hacc & OB (\%) & Caco-2 & BBB & DL & FSAF & TPSA & RBN \\
\hline Solasonine & 884.19 & 0.02 & 10 & 17 & 25.94 & -2.32 & -3.43 & 0.06 & 0.2 & 258.71 & 8 \\
\hline
\end{tabular}

Abbreviations: Caco-2, Caco-2 permeability; OB, oral bioavailability; DL, drug-likeness; BBB, blood-brain barrier.

predicted upstream regulators (Table S5). The top 30 predicted upstream regulators are listed in Figure 1(e). The ERK cascade was the most significant upstream regulator targeting 11 genes, followed by 9 EGFR targeting genes. Notably, both the upstream regulators ERK and EGFR and the other five upstream regulators AKT, KDR, MET, SMAD2, and SMAD3 were involved in the causal regulatory network associated with NRP1, mainly functioning in tissue migration, angiogenesis, growth, proliferation, hypoxia, MAPK signaling, and protein tyrosine kinase (PTK) activity (Figure 1(f)). In addition, the potential hepatotoxicity, nephrotoxicity, and cardiotoxicity of solasonine were predicted by toxicity analysis using IPA (Figure $1(\mathrm{~g})$ and Table S6).

3.3. Target Identification and Analysis for BC. By searching the databases of GeneCards, OMIM, TTD, PharmGKB, and DrugBank, we obtained a total of 9288 genes related to the occurrence and development of BC after eliminating the duplicated ones (Figure 2(a)). Integration of the potential targets regulated by solasonine, and the genes associated with BC revealed that 191 genes overlapped (Figure 2(b) and Table S7) and could be candidate therapeutic targets of solasonine in BC.

3.4. GO and KEGG Pathway Analyses of Gene Targets for Solasonine in BC. KEGG pathway enrichment analysis showed that these potential therapeutic targets of solasonine for BC were significantly enriched in three pathways (adjusted $P$-value $\leq 0.05)$, including proteoglycans in cancer, HIF-1 signaling pathway, and Yersinia infection. In addition, the results of GO term analysis indicated that a total of 692,16 , and 48 terms (adjusted $P$-value $\leq 0.05$ ) were remarkably enriched in biological process (BP), cellular component (CC), and molecular function (MF), respectively (Table S8). The top three KEGG pathways, as well as BP, CC, and $M F$ terms, sorted by adjusting $P$-values in increasing order, are presented in a bar graph (Figure 2(c)), showing a significant enrichment of organ growth (BP), transcription factor complex (CC), and protein tyrosine kinase activity (MF). The gene-pathway/GO terms network showed the interactions among the gene targets for solasonine and the top significant enriched pathways/GO terms (Figure 2(d)).

3.5. Solasonine Affects BC Cell Viability and Inhibits Cell Proliferation. The CCK8 assay was performed to determine the effect of solasonine on the viability and growth of BC cells. As illustrated in Figure 3(a), no obvious inhibitory effect on cell viability was observed in T24 cells before treatment with solasonine at $60 \mu \mathrm{M}$ for $48 \mathrm{~h}$. The 5637 cells showed improved tolerance until $90 \mu \mathrm{M}$ solasonine was used. Thus, in subsequent experiments, 50 and $80 \mu \mathrm{M}$ solasonine doses were selected for T24 and 5637 cells, respectively. Proliferation curves showed that, compared with the control group, cell proliferation was markedly inhibited by solasonine in a time-dependent manner (Figure 3(b)). These results demonstrated that solasonine may function as a tumor suppressor in BC cells. 

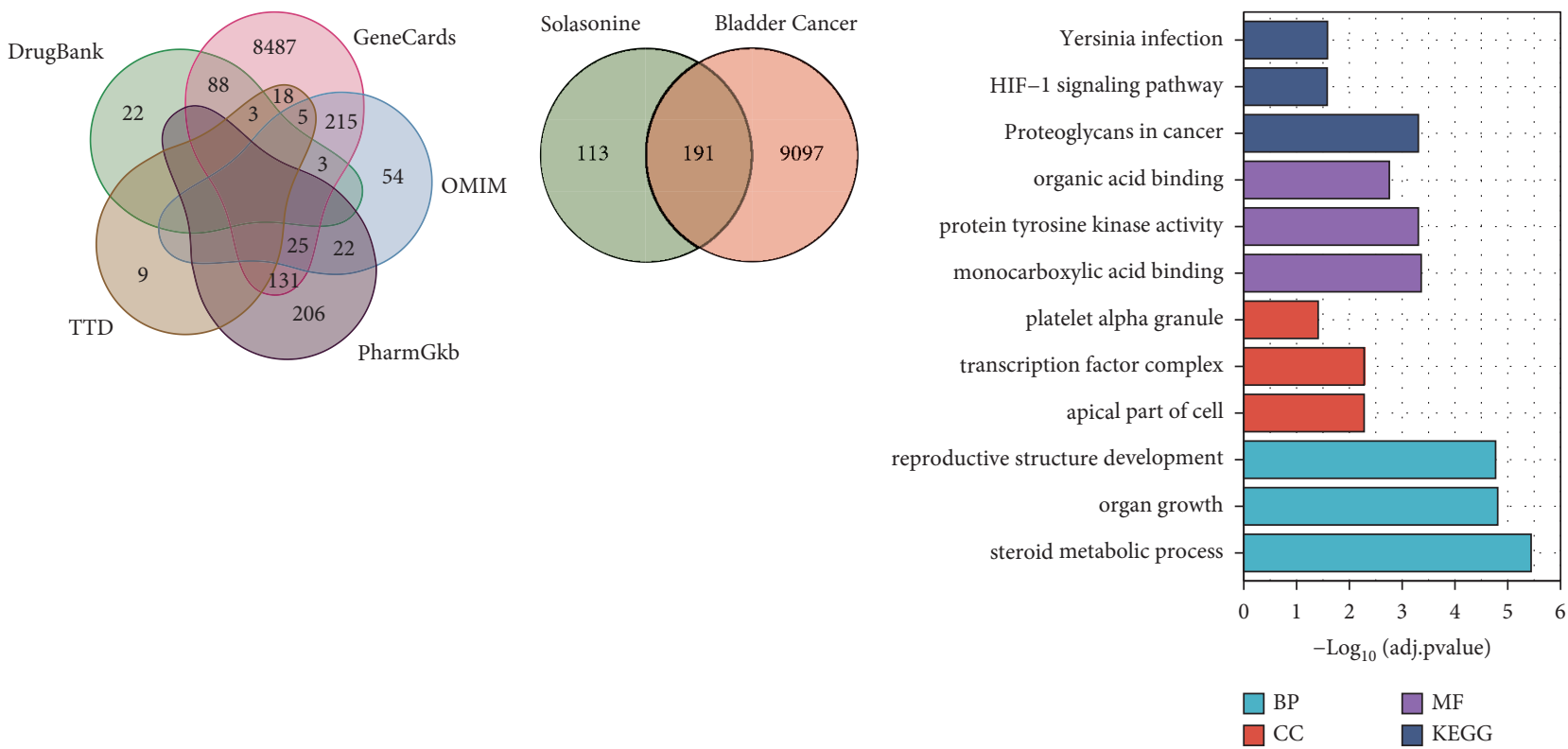

(a)

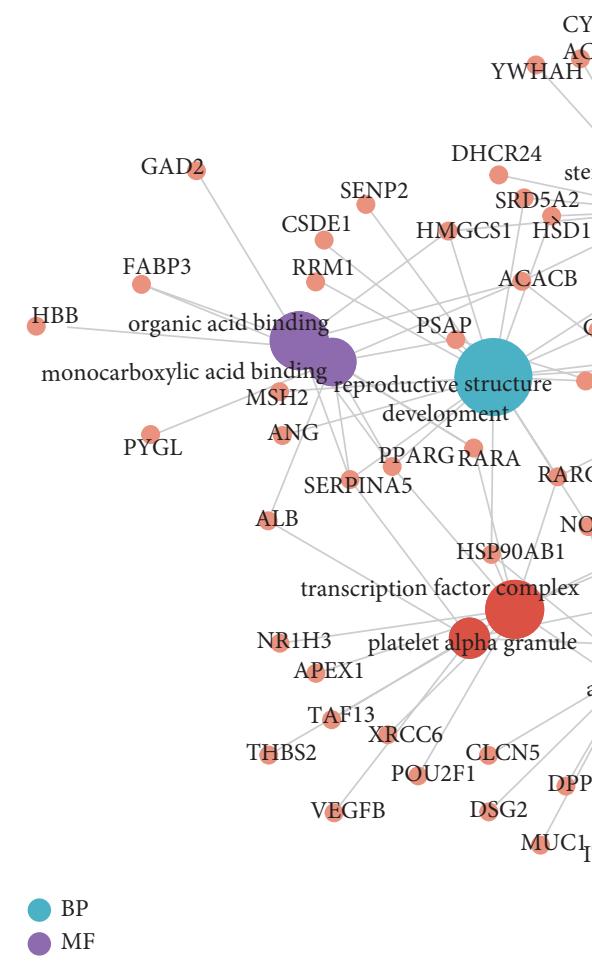

(b) CYP2E1 CYP7A1 CYP17A1 SERPINA6 SULT1A GGPS

(c)

(c)

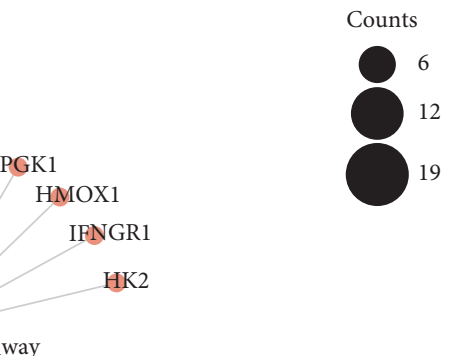

SHH HIF-1 signaling pathway ALK

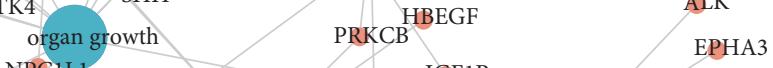

NPC1L1 ERBB4 IGF1R WEE1

NOTCH1 Proteoglycans in cancer FAS protein tyrosine kinase activity

YY1 Proteoglycansineancer TLR4 ITGB3
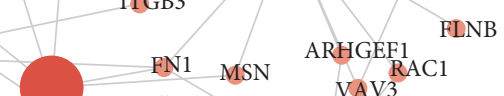

RDNB

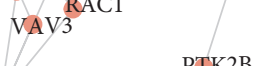

PTK2B

HCK

MATK

BTK

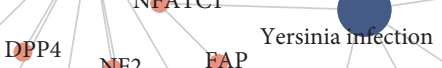

UC1 ${ }_{\text {ITPK1 SLC5A1 }}^{\text {TJP1 }}$ FCGR2A IL2

$\mathrm{CC}$

KEGG

(d)

Figure 2: Target identification and functional enrichment analysis of potential target genes of solanine in bladder cancer. (a) Venn graph showing the overlapping and specific genes associated with BC in GeneCards, OMIM, TTD, PharmGKB, and DrugBank databases. (b) Venn graph showing the overlapping and specific genes by integration of the potential targets regulated by solasonine and genes associated with BC. (c) Bar graph showing the top three significantly enriched KEGG pathways and GO terms (BP, CC, and MF), sorted by adjusted $P$-values in increasing order. (d) Gene-pathway/GO terms network showing interactions among gene targets for solasonine and the top significant enriched pathways/GO terms.

3.6. Solasonine Promotes BC Cell Apoptosis and Induces Cell Cycle Arrest. To investigate the possible mechanism of solasonine on suppressing cell proliferation, we evaluated cell apoptosis and cell cycle on BC cells. Compared with the control group, the proportion of apoptotic cells dramatically increased after solasonine treatment (Figure 3(c)). Flow cytometry analysis of the cell cycle showed that solasonine induced a significant decrease in the percentage of cells in the G0/G1 phase and an accumulation of cells arrested in the $S$ phase, but no obvious alteration in the G2/M phase 

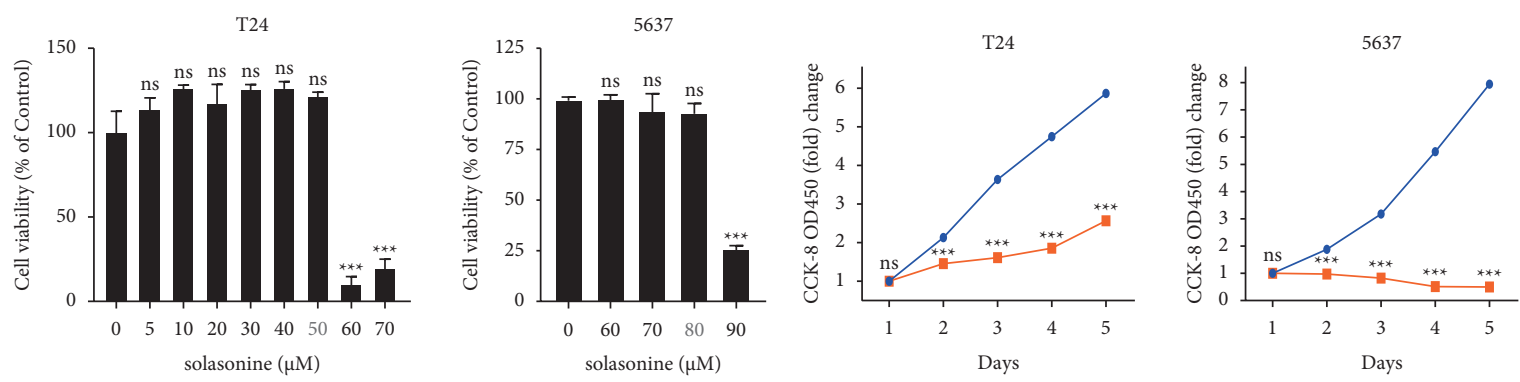

$$
\begin{aligned}
& \longrightarrow \text { NC } \\
& \rightarrow \text { solasonine }
\end{aligned}
$$$$
\rightarrow \text { NC }
$$$$
\neg \text { solasonine }
$$

(a)

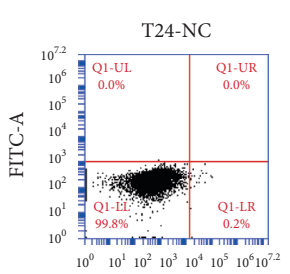

APC-A

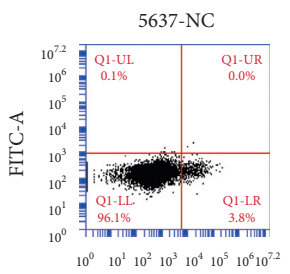
APC-A
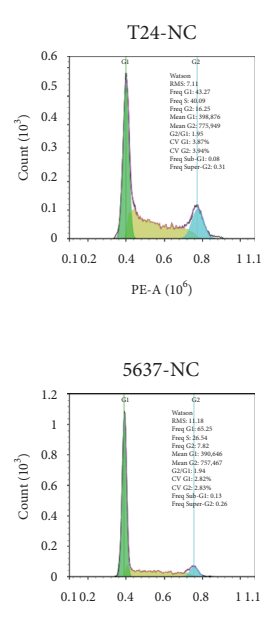

PE-A $\left(10^{6}\right)$
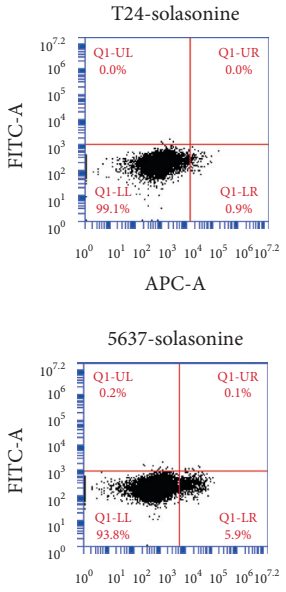

APC-A

(c)
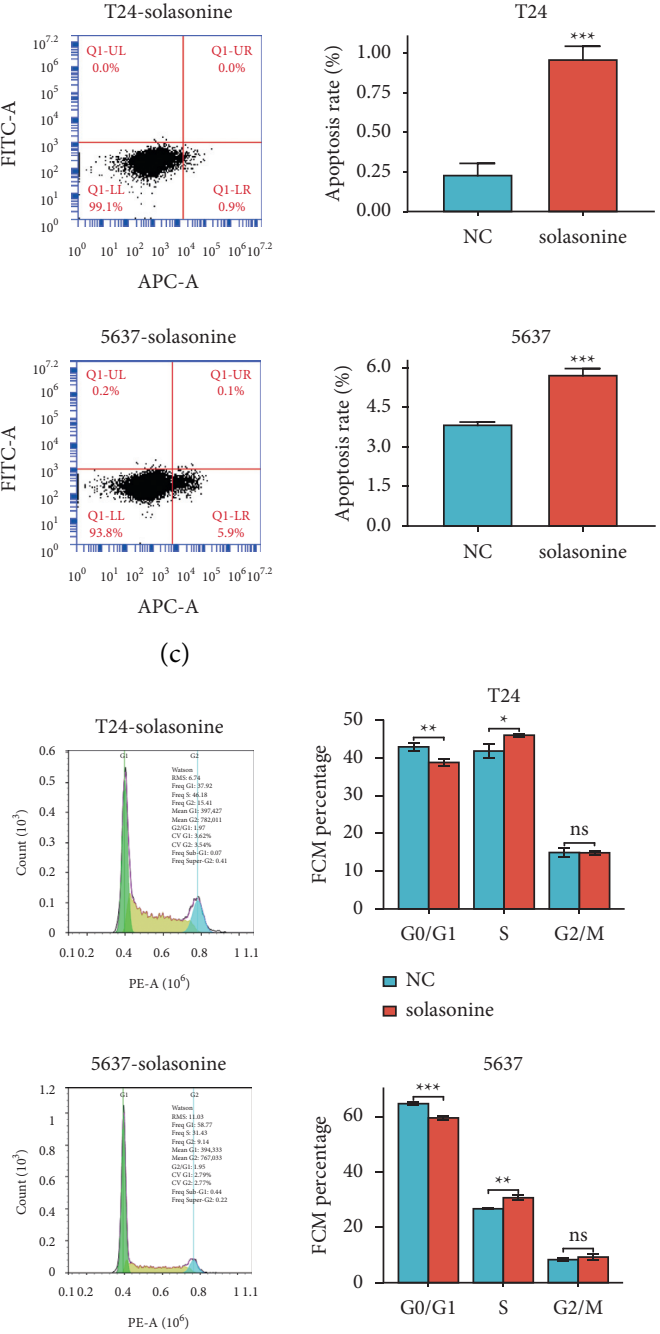

ㅁ solasonine

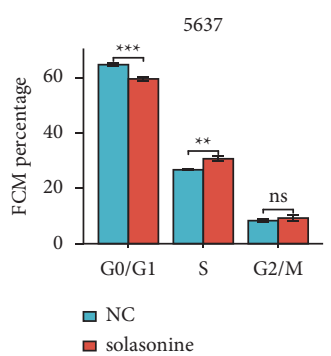

(d)

Figure 3: Continued. 


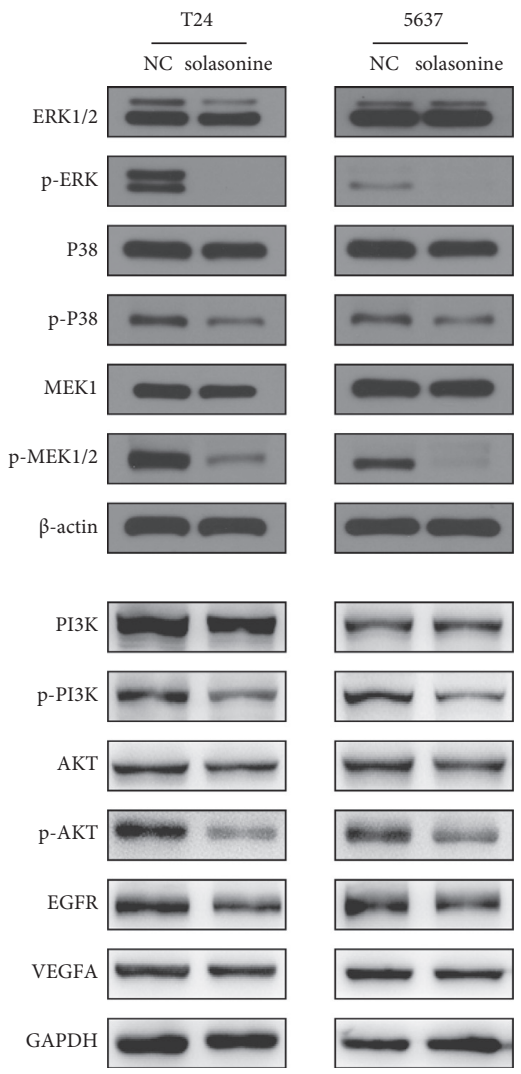

(e)

FIGURE 3: Effects of solasonine on cell proliferation, apoptosis, cell cycle arrest, and the expression of genes in tumor-related pathways in BC cells. (a) T24 and 5637 cells were treated with various concentrations of solasonine for $48 \mathrm{~h}$, and the cell viability was evaluated by CCK8 assay. The doses of 50 and $80 \mu \mathrm{M}$ solasonine were selected for T24 and 5637 cells in subsequent experiments, respectively. (b) CCK8 assay revealed that solasonine significantly reduced the proliferation of $\mathrm{BC}$ cells in a time-dependent manner. (c) Apoptosis assay and quantitation of apoptotic cells showed that solasonine significantly promoted apoptosis in BC cells. (d) Flow cytometric analysis showed that solasonine affected cell cycle progression. (e) Western blot showed changes of molecules in the MAPK and PI3K/AKT signaling pathways, EGFR and VEGFA protein expression in T24 and 5637 BC cells after solasonine treatment. Three independent experiments were conducted for each assay, and data are presented as the mean \pm standard error of the mean. ${ }^{*} P<0.05,{ }^{* *} P<0.01$, and ${ }^{* * *} P<0.001$ vs. the control group.

(Figure 3(d)). The results revealed that solasonine inhibited $\mathrm{BC}$ cell proliferation by promoting apoptosis and inhibiting the G0/G1 and S phase transitions.

\subsection{Solasonine Inhibits MAPK and PI3K/AKT Pathways.} We performed Western blot to confirm the prediction of canonical pathway analysis by IPA. It was found that the MAPK and PI3K/AKT signaling pathways were involved in the antitumor activity of solasonine. As shown in Figure 3(e), solasonine obviously decreased the phosphorylation of ERK, P38, and MEK1/2 in T24 and 5637 cells, suggesting a close relationship between the inhibition of ERK/MAPK, P38/ MAPK, and PI3K/AKT signaling pathways with solasonine treatment. In addition, the expression of EGFR and VEGF, two predicted upstream regulators for solasonine identified by IPA analysis, did not differ significantly after solasonine treatment.

3.8. Analysis of Molecular Docking and MDS. Molecular docking analysis suggested that solasonine strongly interacts with the catalytic pocket of NRP1. Binding affinity was measured in $\mathrm{kcal} / \mathrm{mol}$, with a smaller affinity indicating stronger binding. An affinity of $<-7.00 \mathrm{kcal} / \mathrm{mol}$ represents a satisfactory binding strength between the ligand and receptor. It was found solasonine could form hydrogen bonds with several residues and hydrophobic effect with TYR297 bound to NRP1 (affinity $=-8.1 \mathrm{kcal} / \mathrm{mol}$ ), which could stabilize the NRP1 protein and mediate the anchorage of solasonine in the binding site of NRP1 to form a stable complex (Figures 4(a)-4(b)). Further MDS analysis confirmed the docking stability of the NRP1-solasonine complex. The structural variations of NRP1-solasonine complex were examined by RMSD values from 0 to $200 \mathrm{~ns}$, showing a steady increase at the beginning and a stable state throughout the simulation with an average RMSD value of $0.26 \mathrm{~nm}$ (Figure 4(c)). The fluctuation of the binding site residues in the NRP1-solasonine docking complex was reflected by RMSF values, which showed a stable docking pose with an average RMSF value of $0.07 \mathrm{~nm}$ (Figure $4(\mathrm{~d})$ ). Moreover, the $\mathrm{Rg}$ value of the complex was in the range of 1.42 and $1.47 \mathrm{~nm}$ in MDS (Figure 4(e)), and the hydrogen bonds were varied between 0 and 8 (Figure 4(f)). 


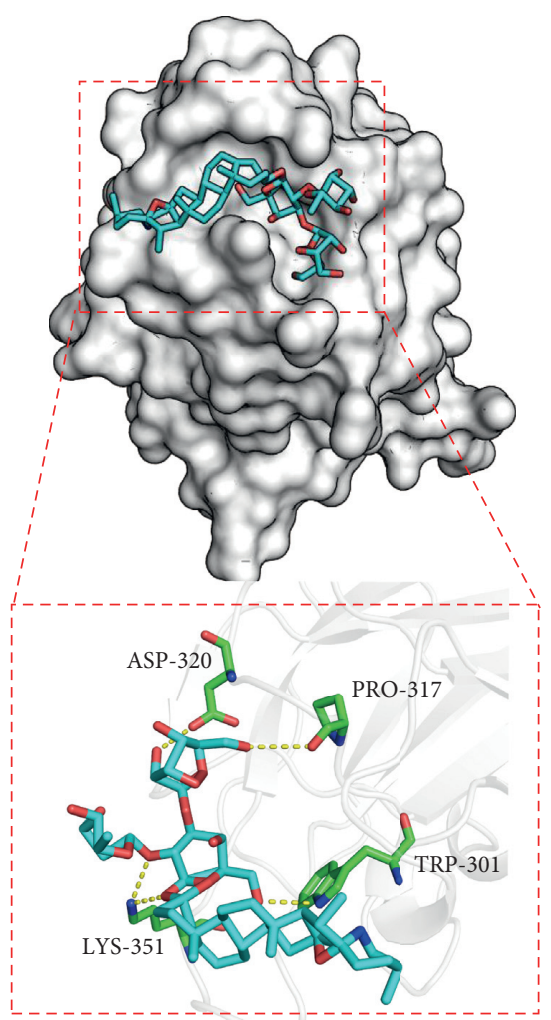

(a)

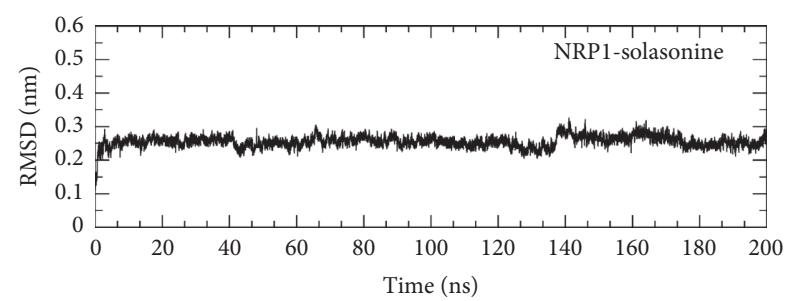

(c)

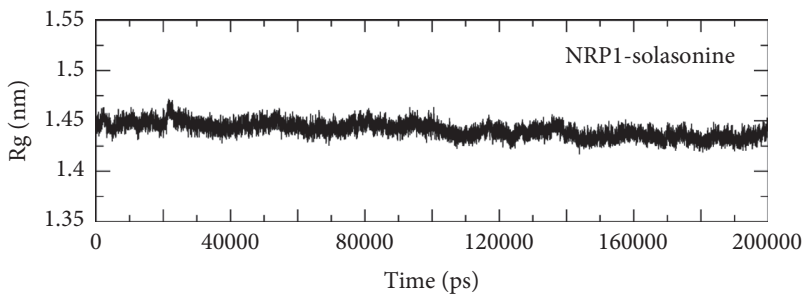

(e)
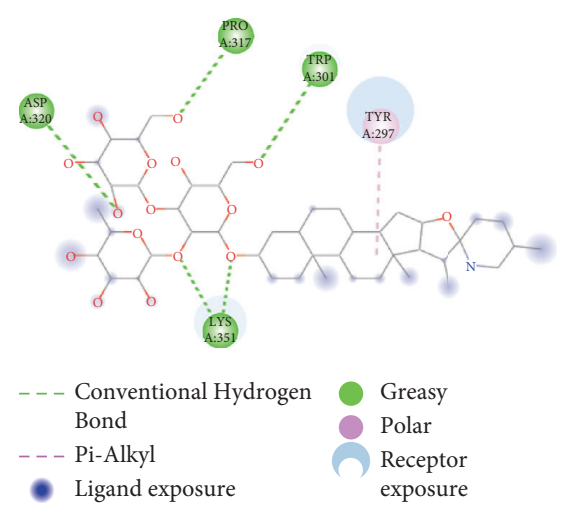

(b)

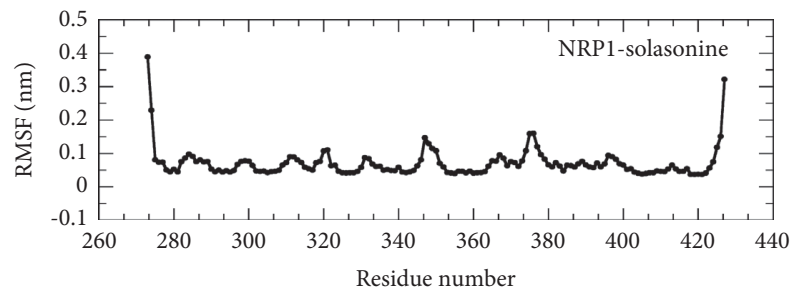

(d)

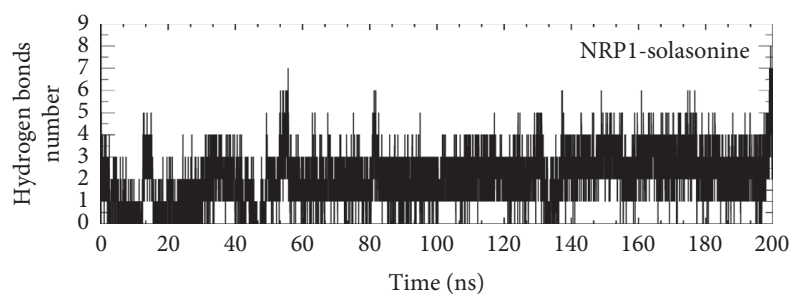

_ Hydrogen bond

(f)

FIgure 4: Continued. 


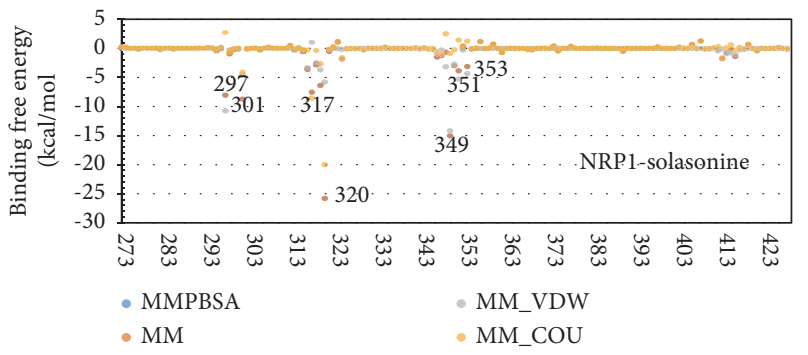

(g)

FIGURE 4: Molecular docking, MDS, and binding free energy calculation for NRP1 docking with solasonine. Molecular docking modes for NRP1 docking with solasonine in (a) 3D and (b) 2D images. Molecular surface representation of NRP1 (white) was shown as the docking pose and interaction of solasonine (blue stick model) with the primary pocket. (c) Plot of root-mean-square deviation (RMSD) values during 200 ns MDS of NRP1 docking with solasonine. (d) Plot of root-mean-square fluctuation (RMSF) values during $200 \mathrm{~ns}$ MDS. (e) Plot of radius of gyration (Rg) values during $200 \mathrm{~ns}$ MDS. (f) Plot of hydrogen bond number during $200 \mathrm{~ns}$ MDS of NRP1 docking with solasonine. (g) Plot of binding free energy simulation of NRP1 docking with solasonine.

3.9. Binding Free Energy Calculation. The binding free energy for the NRP1-solasonine complex was calculated to be $-97.744 \mathrm{kcal} / \mathrm{mol}$ (Figure $4(\mathrm{~g})$ and Table S9). Consistent with the molecular docking results, it is also evident from the binding free energies that TYR297, TRP301, PRO317, ASP320, and LYS351 residues mainly contributed to mediating anchorage with solasonine to form a stable NRP1solasonine docking. The binding free energy was dominated by van der Waals and Coulombic electrostatic interaction energies. Solasonine binds most tightly with the ASP320 residue of NRP1 with a binding free energy of $-25.80 \mathrm{kcal} /$ mol mainly by Coulombic interaction energy, and the next most tightly bound residue was THR349, with a binding free energy of $-15.04 \mathrm{kcal} / \mathrm{mol}$ mainly by van der Waals interaction energy, both of which were the main binding favorable for stabilizing the NRP1-solasonine complex.

3.10. Solasonine Inhibits NRP1 Protein Expression. Solasonine treatment resulted in a significant inhibitory effect on NRP1 protein expression (Figure 5(a)), but no significant difference was detected in NRP1 mRNA expression levels (Figure 5(b)). The NRP1 structure was shown, and the extracellular b1 domain was predicted to bind to solasonine (Figure 5(c)).

3.11. Solasonine-NRP1 Pathway Constructed in the Present Study. We further constructed hypothetical solasonineNRP1 interactions, showing that extracellular solasonine binds to NRP1 at the cell membrane and thereby prevents the binding of VEGFA/VEGFR2 and EGFR to NRP1, resulting in the inhibition of the subsequent activation of downstream pathways, including ERK/MAPK, P38/MAPK, and PI3K/AKT pathways; in addition, intracellular solasonine bound to NRP1 to block the membrane localization of NRP1 and then downregulated NRP1 protein expression by facilitating its degradation (Figure 6). We hypothesized that solasonine might dock NRP1 to induce apoptosis and inhibit proliferation of BC cells.

\section{Discussion}

As single-pass transmembrane, non-tyrosine kinase surface glycoproteins, neuropilins (NRPs), are unique to vertebrates and highly conserved across species [50]. NRPs include two isoforms, NRP1 and NRP2. NRP1 was discovered in 1987 and was originally identified as a receptor in neuronal and endothelial cells and is essential for regulating neural and vascular development [22]. In recent years, high expression of NRP1 was found in other types of cells, such as immune cells, osteoblasts, adipocytes, hepatic stellate cells, and glomerular stromal cells. Increasing evidence on the involvement of NRP1 in cancer and immune processes has triggered research interests worldwide [22, 50-52]. NRP1 is considered a potential therapeutic target in the novel coronavirus disease 2019 [53, 54]. In addition, a growing body of evidence on NRP1-mediated immune modulation suggests that NRP1 is a novel immune checkpoint and immunotherapeutic target that may provide a durable anticancer immunity and help maintain long-term remission in cancer patients, either alone or in combination with current immunotherapeutic strategies $[21,22]$. Therefore, the translation of NRP1 into therapeutic interventions is promising.

We previously showed that NRP1 was upregulated in BC, and NRP1 silencing could induce cell apoptosis and suppress proliferation, invasion, metastasis, and angiogenesis in BC cells. We also found that inactivation of the MAPK signaling pathway was involved in mediating the anti-BC effects of NRP1 [24]. In this study, we identified potential genes targeted by solasonine, followed by functional enrichment analysis. According to IPA and KEGG pathway analyses, the majority of these enrichment functions are related to tumorigenesis and progression, including the HIF1 signaling pathway, ERK/MAPK pathway, P38/MAPK pathway, PI3K/AKT pathway, and PTK activity. The Western blot experiment further demonstrated that the activation of ERK/MAPK, P38/MAPK, and PI3K/AKT pathways could be suppressed after solasonine treatment in BC cells. It has been reported that the NRP1 promoter can directly bind to HIF- $1 \alpha$ [55], which is a critical tumor driver 


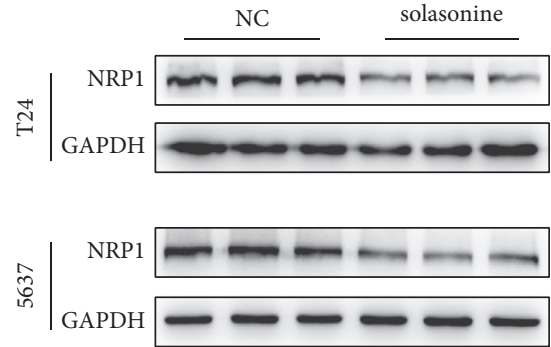

(a)

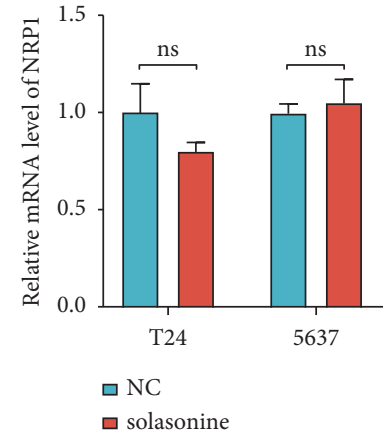

(b)

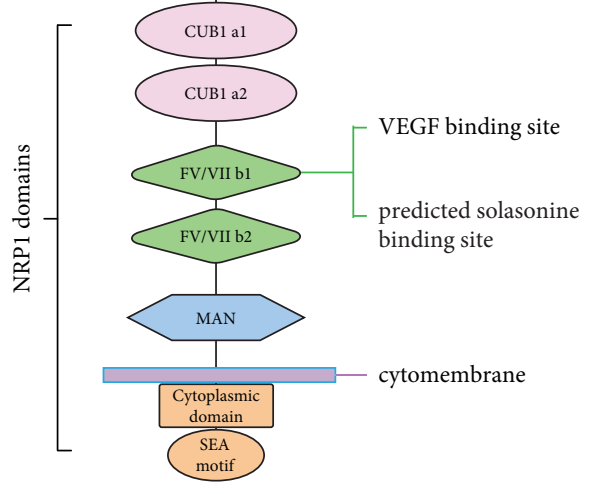

(c)

FIGURE 5: Effect of solasonine on NRP1 expression. (a) Solasonine inhibited NRP1 protein expression level. (b) Solasonine did not affect NRP1 mRNA expression. (c) The structure of NRP1 domains and the extracellular b1 domain was predicted to bind with solasonine.

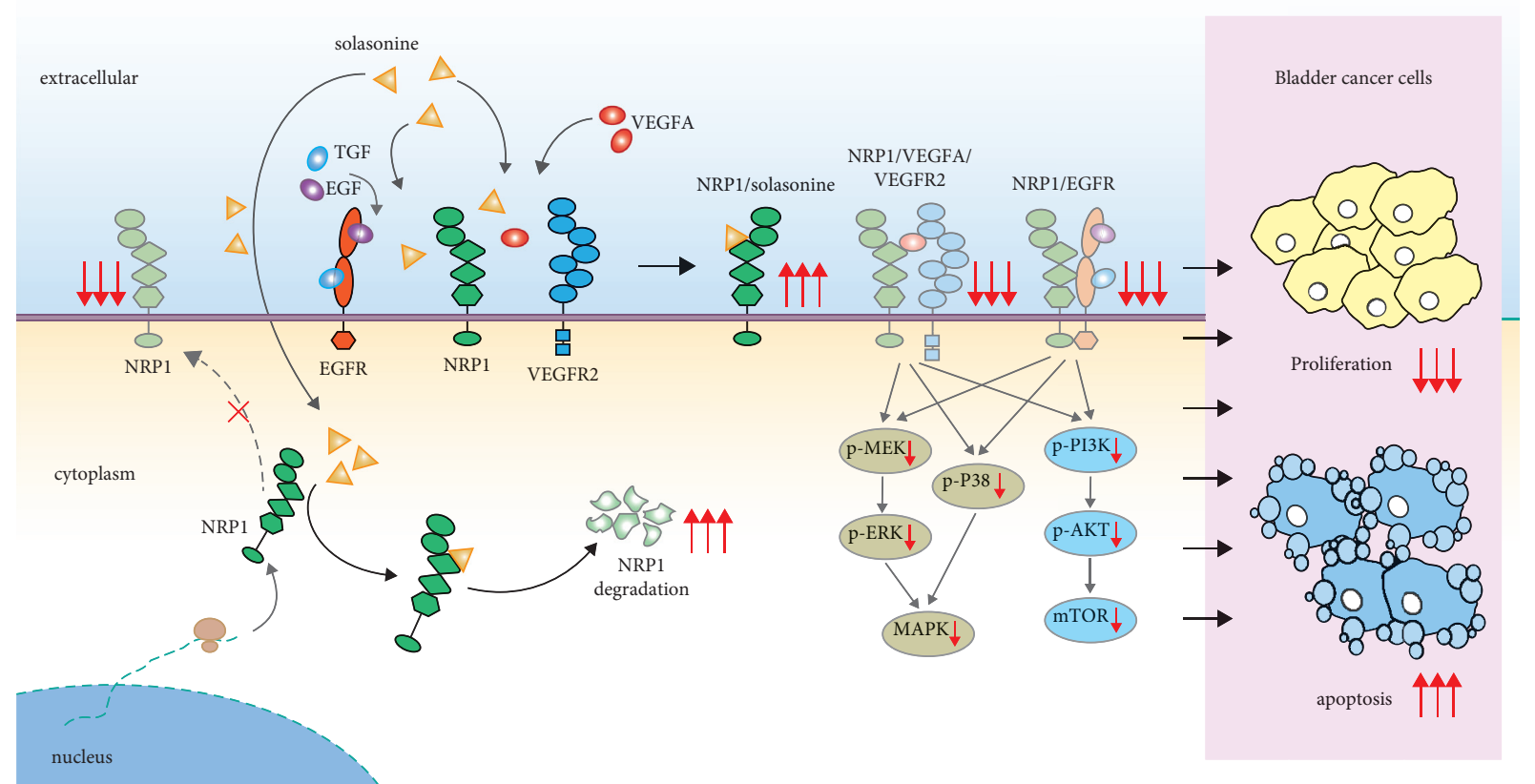

FIGURE 6: Solasonine-NRP1 pathway constructed in this study. The hypothetical solasonine-NRP1 interactions with subsequent inhibition of downstream pathways.

involved in PI3K/AKT regulation and can facilitate the upregulation of both HIF- $1 \alpha$ and PI3K/AKT through a positive feedback loop [56]. Overexpression of HIF-1 could induce upregulation of multiple growth factors, such as VEGF, PDGF, and EGF, that could in turn activate the PI3K/ AKT pathway via receptor tyrosine kinases (RTKs) and subsequently result in the activation of the mammalian target of rapamycin (mTOR) complex to promote HIF-1 upregulation. It is generally accepted that ERK/MAPK is a pro-proliferation and antiapoptosis factor $[57,58]$, and P38/ MAPK is a proliferation promoter in tumors [59]. This observation is consistent with our experimental findings in $\mathrm{BC}$ cells treated with solasonine.

According to the causal regulatory network analysis by IPA, NRP1 was identified as a master upstream regulator involved in solasonine treatment. We then detected the protein and mRNA expression levels of NRP1 in BC cells treated with solasonine. The results showed that treatment with solasonine suppressed NRP1 protein expression but had no effect on NRP1 mRNA expression. Further molecular docking analysis indicated that solasonine could directly target the NRP1 protein with a reasonable docking conformation. The stability and reliability of the tight binding results were validated by the binding free energy of the docked complex calculations through molecular dynamics simulations. These findings suggest that solasonine is not responsible for the transcriptional regulation of NRP1 but may downregulate the stability of NRP1 protein by direct binding to alter the molecular conformation of NRP1. In general, as a membrane protein, NRP1 needs to be inserted into the cell membrane to exert biological functions. By binding to NRP1, solasonine might inhibit the membrane 
localization of NRP1 and provoke its cytoplasmic retention, leading to its degradation in cells. This mechanism could be among the contributors to NRP1 protein downregulation, which requires further experimental verification.

The NRP1 protein consists of a long N-terminal extracellular domain, followed by a transmembrane region and a short cytosolic tail of 43-44 amino acids [22]. Its extracellular domain contains five subdomains, namely, a1, a2, b1, b2, and c, each of which mediates the interaction of NRP1 with different molecules and cells [50]. The b1 domain is the main functional domain responsible for NRP1 binding to the novel coronavirus disease 2019 receptors $[53,54]$ and multiple growth factors and receptors, most notably VEGFA and VEGFR2 [60]. The NRP1b1 domain is also a target for the design of many smallmolecule agents. NRP1 binds to VEGFA and VEGFR2 simultaneously to form an NRP1/VEGFA/VEGFR2 complex, eliciting activation of downstream signal transduction pathways, including ERK/MAPK, P38/MAPK, and PI3K/ AKT pathways $[22,50,60,61]$. Upstream regulator analysis by IPA identified both VEGF and EGFR as upstream regulators of solasonine-targeted genes. Current evidence indicates that the extracellular NRP1 domain could also bind to EGFR, which is essential for activating the EGFR signaling cascade upon EGF or TGF-a stimulation [62]. Meanwhile, following NRP1 silencing, the ability of ligandbound EGFR to cluster on the cytomembrane and AKT pathway activity was severely impaired [62]. In our study, the protein expression levels of both EGFR and VEGF were not remarkably affected by solasonine treatment. These findings imply that solasonine interferes with NRP1 binding, which only affects the transduction of EGFR and VEGF signaling cascades, but not their expression.

Based on these results, we inferred that solasonine binding with NRP1 on the cell membrane could prevent the formation of NRP1/VEGFA/VEGFR2 and NRP1/EGFR complexes, resulting in the inhibition of downstream pathways, including ERK/MAPK, P38/MAPK, and PI3K/ AKT pathways; meanwhile, intracellular solasonine could inhibit the membrane localization of NRP1 and provoke its cytoplasmic retention, facilitating the degradation of NRP1 protein in the cytoplasm. The subsequent dual effects induced by the binding of solasonine to NRP1 extracellularly and intracellularly could be potential contributors to the solasonine-dependent antiproliferative and proapoptotic effects on BC cells.

There were some limitations in this study. First, although the target prediction for solasonine against $\mathrm{BC}$ was performed in several online databases, it lacked accuracy and specificity compared with mass spectrometry and sequencing experiments. Second, we did not construct the silenced and overexpressed NRP1 groups to observe the anticancer effect of solasonine, which would weaken the evidence that solasonine targeting NRP1 induces apoptosis and inhibits proliferation in BC cells. Finally, immunoprecipitation experiments should be performed to confirm the binding of solasonine to NRP1 and to validate the mechanism of solasonine activity against $\mathrm{BC}$ observed in this study. These issues can be addressed in subsequent experiments. Our findings suggest a potential novel mechanism by which solasonine inhibits the growth of BC cells and indicate an important role of NRP1 in the activity of solasonine. Considering the widespread application of $S$. nigrum in various disorders [12], exploring the biological mechanism underlying the activity of solasonine could help reduce the incidence of toxic side effects and advance the development of precision or personalized therapies at a low cost.

\section{Conclusions}

In this study, the mechanisms and candidate targets of solasonine in the treatment of $\mathrm{BC}$ were examined using bioinformatics analyses and verified using experimental models. Molecular docking predicted that solasonine could directly bind to NRP1. The inhibition of NRP1 protein expression was observed in $\mathrm{BC}$ cells following solasonine treatment. We suggested that solasonine could inhibit the MAPK and PI3K/AKT pathways by preventing the formation of NRP1/VEGFA/VEGFR2 and NRP1/EGFR complexes on the membrane surface, inducing NRP1 protein degradation by restricting its membrane localization. The dual effects induced by solasonine-NRP1 binding extracellularly and intracellularly could be potential contributors to the antitumor effect of solasonine.

\section{Data Availability}

The data that support the findings of this study are available from the corresponding author upon reasonable request.

\section{Conflicts of Interest}

The authors declare that they have no conflicts of interest.

\section{Authors' Contributions}

Yang Dong, Lin Hao, Zhen-duo Shi, and Kun Fang contributed equally to this work.

\section{Acknowledgments}

This work was supported by the National Natural Science Foundation of China (grant numbers 82004110 and 81774089); the Medical Innovation Team Project of Jiangsu Province (grant number CXTDA-2017-48); the Jiangsu Provincial Key Research and Development Program (grant numbers BE2020758 and BE2019637); the Key Project of Xuzhou Science and Technology (grant numbers KC19075 and KC18036); and the Xuzhou Youth Medical Science and Technology Innovation Project (XWKYSL20210277).

\section{Supplementary Materials}

Table S1. Potential target genes of solasonine. Table S2. Canonical pathway analysis by IPA for solasonine. Table S3. Disease and functional analysis by IPA for solasonine. Table S4. Upstream analysis by IPA for solasonine. Table S5. Causal regulatory network analysis by IPA for solasonine. Table S6. Toxicity analysis by IPA for solasonine. Table S7. Potential therapeutic targets of solasonine for BC. Table S8. 
KEGG pathway and GO analyses for genes of solasonine targeting BC. Table S9. Binding free energy of NRP1 docking with solasonine. (Supplementary Materials)

\section{References}

[1] H. Sung, J. Ferlay, R. L. Siegel et al., "Global cancer statistics 2020: GLOBOCAN estimates of incidence and mortality worldwide for 36 cancers in 185 countries," CA: A Cancer Journal for Clinicians, vol. 71, no. 3, pp. 209-249, 2021.

[2] R. L. Siegel, K. D. Miller, H. E. Fuchs, and A. Jemal, "Cancer statistics, 2022," CA: A Cancer Journal for Clinicians, vol. 72, no. 1, pp. 7-33, 2022.

[3] S. S. Chang, S. A. Boorjian, R. Chou et al., "Diagnosis and treatment of non-muscle invasive bladder cancer: AUA/SUO guideline," The Journal of Urology, vol. 196, no. 4, pp. 1021-1029, 2016.

[4] J. B. Aragon-Ching, R. P. Werntz, A. L. Zietman, and G. D. Steinberg, "Multidisciplinary management of muscleinvasive bladder cancer: current challenges and future directions," Am Soc Clin Oncol Educ Book, vol. 38, pp. 307-318, 2018.

[5] E. I. Heath and J. E. Rosenberg, "The biology and rationale of targeting nectin-4 in urothelial carcinoma," Nature Reviews Urology, vol. 18, no. 2, pp. 93-103, 2021.

[6] A. T. Lenis, P. M. Lec, K. Chamie, and M. Mshs, "Bladder cancer," JAMA, vol. 324, no. 19, pp. 1980-1991, 2020.

[7] V. G. Patel, W. K. Oh, and M. D. Galsky, "Treatment of muscle-invasive and advanced bladder cancer in 2020," CA: A Cancer Journal for Clinicians, vol. 70, no. 5, pp. 404-423, 2020.

[8] D. Schmid, C. G. Park, C. A. Hartl et al., "T cell-targeting nanoparticles focus delivery of immunotherapy to improve antitumor immunity," Nature Communications, vol. 8, no. 1, p. $1747,2017$.

[9] V. Vashistha, D. I. Quinn, T. B. Dorff, and S. Daneshmand, "Current and recent clinical trials for perioperative systemic therapy for muscle invasive bladder cancer: a systematic review," BMC Cancer, vol. 14, p. 966, 2014.

[10] L. Tran, J.-F. Xiao, N. Agarwal, J. E. Duex, and D. Theodorescu, "Advances in bladder cancer biology and therapy," Nature Reviews Cancer, vol. 21, no. 2, pp. 104-121, 2021.

[11] D. Hanahan, "Hallmarks of cancer: new dimensions," Cancer Discovery, vol. 12, no. 1, pp. 31-46, 2022.

[12] R. Jain, A. Sharma, S. Gupta, I. P. Sarethy, and R. Gabrani, "Solanum nigrum: current perspectives on therapeutic properties," Alternative Medicine Review: A Journal of Clinical Therapeutic, vol. 16, no. 1, pp. 78-85, 2011.

[13] Y. Dong, L. Hao, K. Fang et al., "A network pharmacology perspective for deciphering potential mechanisms of action of Solanum nigrum L. in bladder cancer," BMC Complementary Medicine and Therapies, vol. 21, no. 1, p. 45, 2021.

[14] S. E. Milner, N. P. Brunton, P. W. Jones, N. M. O’ Brien, S. G. Collins, and A. R. Maguire, "Bioactivities of glycoalkaloids and their aglycones from Solanum species," Journal of Agricultural and Food Chemistry, vol. 59, no. 8, pp. 3454-3484, 2011.

[15] C. C. Munari, P. F. de Oliveira, J. C. L. Campos et al., "Antiproliferative activity of Solanum lycocarpum alkaloidic extract and their constituents, solamargine and solasonine, in tumor cell lines," Journal of Natural Medicines, vol. 68, no. 1, pp. 236-241, 2014.

[16] F. Tutin and H. W. B. Clewer, "LVII.-The constituents of Solanum angustifolium: isolation of a new gluco-alkaloid, solangustine," Journal of the Chemical Society Transactions, vol. 105, no. 0, pp. 559-576, 1914.

[17] H. Zhang, F. Tian, P. Jiang et al., "Solasonine suppresses the proliferation of acute monocytic leukemia through the activation of the AMPK/FOXO3A Axis," Frontiers Oncology, vol. 10, p. 614067, 2020.

[18] Z. Liu, C. Ma, X. Tang et al., "The reciprocal interaction between LncRNA CCAT1 and miR-375-3p contribute to the downregulation of IRF5 gene expression by solasonine in HepG2 human hepatocellular carcinoma cells," Frontiers in Oncology, vol. 9, p. 1081, 2019.

[19] M. Q. Pham, T. H. V. Tran, Q. L. Pham, and J. E. Gairin, "In silico analysis of the binding properties of solasonine to mortalin and p53, and in vitro pharmacological studies of its apoptotic and cytotoxic effects on human HepG2 and Hep3b hepatocellular carcinoma cells," Fundamental \& Clinical Pharmacology, vol. 33, no. 4, pp. 385-396, 2019.

[20] X. Wang, S. Zou, Y. L. Lan, J. S. Xing, X. Q. Lan, and B. Zhang, "Solasonine inhibits glioma growth through anti-inflammatory pathways," American Journal of Tourism Research, vol. 9, no. 9, pp. 3977-3989, 2017.

[21] N. Acharya and A. C. Anderson, "NRP1 cripples immunological memory," Nature Immunology, vol. 21, no. 9, pp. 972-973, 2020.

[22] C. A. Chuckran, C. Liu, T. C. Bruno, C. J Workman, and D. A Vignali, "Neuropilin-1: a checkpoint target with unique implications for cancer immunology and immunotherapy," Journal for immunotherapy of cancer, vol. 8, no. 2, 2020.

[23] C. Liu, A. Somasundaram, S. Manne et al., "Neuropilin-1 is a $\mathrm{T}$ cell memory checkpoint limiting long-term antitumor immunity," Nature Immunology, vol. 21, no. 9, pp. 1010-1021, 2020.

[24] Y. Dong, W.-m. Ma, Z.-d. Shi et al., "Role of NRP1 in bladder cancer pathogenesis and progression," Frontiers in Oncology, vol. 11, Article ID 685980, 2021.

[25] B. Bienfait and P. Ertl, "JSME: a free molecule editor in JavaScript," Journal of Cheminformatics, vol. 5, no. 1, p. 24, 2013.

[26] J. Ru, P. Li, J. Wang et al., "TCMSP: a database of systems pharmacology for drug discovery from herbal medicines," Journal of Cheminformatics, vol. 6, no. 1, p. 13, 2014.

[27] X. Liu, S. Ouyang, B. Yu et al., "PharmMapper server: a web server for potential drug target identification using pharmacophore mapping approach," Nucleic Acids Research, Web Server Issue, vol. 38, no. suppl_2, pp. W609-W614, 2010.

[28] X. Wang, Y. Shen, S. Wang et al., "PharmMapper 2017 update: a web server for potential drug target identification with a comprehensive target pharmacophore database," Nucleic Acids Research, vol. 45, no. W1, pp. W356-W360, 2017.

[29] Z.-J. Yao, J. Dong, Y.-J. Che et al., "TargetNet: a web service for predicting potential drug-target interaction profiling via multi-target SAR models," Journal of Computer-Aided Molecular Design, vol. 30, no. 5, pp. 413-424, 2016.

[30] M. J. Keiser, B. L. Roth, B. N. Armbruster, P. Ernsberger, J. J. Irwin, and B. K. Shoichet, "Relating protein pharmacology by ligand chemistry," Nature Biotechnology, vol. 25, no. 2, pp. 197-206, 2007.

[31] G. Stelzer, N. Rosen, I. Plaschkes et al., "The GeneCards suite: from gene data mining to disease genome sequence analyses," Current Protocols in Bioinformatics, vol. 54, pp. 130 33-31 30 33, 2016.

[32] V. A. McKusick, "Mendelian inheritance in man and its online version, OMIM," The American Journal of Human Genetics, vol. 80, no. 4, pp. 588-604, 2007. 
[33] Y. Wang, S. Zhang, F. Li et al., "Therapeutic target database 2020: enriched resource for facilitating research and early development of targeted therapeutics," Nucleic Acids Research, vol. 48, no. D1, pp. D1031-D1041, 2020.

[34] M. Whirl-Carrillo, R. Huddart, L. Gong et al., "An evidencebased framework for evaluating Pharmacogenomics knowledge for personalized medicine," Clinical Pharmacology \& Therapeutics, vol. 110, no. 3, pp. 563-572, 2021.

[35] D. S. Wishart, Y. D. Feunang, A. C. Guo et al., "DrugBank 5.0: a major update to the DrugBank database for 2018," Nucleic Acids Research, vol. 46, no. D1, pp. D1074-D1082, 2018.

[36] C. The Gene Ontology, "Expansion of the gene ontology knowledgebase and resources," Nucleic Acids Research, vol. 45, no. D1, pp. D331-D338, 2017.

[37] M. Kanehisa, M. Furumichi, M. Tanabe, Y. Sato, and K. Morishima, "KEGG: new perspectives on genomes, pathways, diseases and drugs," Nucleic Acids Research, vol. 45, no. D1, pp. D353-D361, 2017.

[38] M. Kanehisa and S. Goto, "KEGG: kyoto encyclopedia of genes and genomes," Nucleic Acids Research, vol. 28, no. 1, pp. 27-30, 2000.

[39] G. Yu, L.-G. Wang, G.-R. Yan, and Q.-Y. He, "DOSE: an R/ Bioconductor package for disease ontology semantic and enrichment analysis," Bioinformatics, vol. 31, no. 4, pp. 608-609, 2015.

[40] G. Yu, L.-G. Wang, Y. Han, and Q.-Y. He, "clusterProfiler: an $\mathrm{R}$ Package for comparing biological themes among gene clusters," OMICS: A Journal of Integrative Biology, vol. 16, no. 5, pp. 284-287, 2012.

[41] D. Warde-Farley, S. L. Donaldson, O. Comes et al., "The GeneMANIA prediction server: biological network integration for gene prioritization and predicting gene function," Nucleic Acids Research, Web Server Issue, vol. 38, no. suppl_2, pp. W214-W220, 2010.

[42] P. Shannon, A. Markiel, O. Ozier et al., "Cytoscape: a software environment for integrated models of biomolecular interaction networks," Genome Research, vol. 13, no. 11, pp. 2498-2504, 2003.

[43] G. M. Morris, R. Huey, W. Lindstrom et al., "AutoDock4 and AutoDockTools4: automated docking with selective receptor flexibility," Journal of Computational Chemistry, vol. 30, no. 16, pp. 2785-2791, 2009.

[44] O. Trott and A. J. Olson, "AutoDock Vina: improving the speed and accuracy of docking with a new scoring function, efficient optimization, and multithreading," Journal of Computational Chemistry, vol. 31, no. 2, pp. 455-461, 2010.

[45] W. Humphrey, A. Dalke, and K. Schulten, "VMD: visual molecular dynamics," Journal of Molecular Graphics, vol. 14, no. 1, pp. 33-38, 1996.

[46] D. Van Der Spoel, E. Lindahl, B. Hess, G. Groenhof, A. E. Mark, and H. J. C. Berendsen, "GROMACS: fast, flexible, and free," Journal of Computational Chemistry, vol. 26, no. 16, pp. 1701-1718, 2005.

[47] N. Homeyer and H. Gohlke, "Free energy calculations by the molecular mechanics Poisson-Boltzmann surface area method," Molecular Informatics, vol. 31, no. 2, pp. 114-122, 2012.

[48] K. Pang, Z. Zhang, L. Hao et al., "The ERH gene regulates migration and invasion in 5637 and T24 bladder cancer cells," BMC Cancer, vol. 19, no. 1, p. 225, 2019.

[49] J. J. Zhang, L. J. Cai, K. Pang et al., "Paeonol inhibits proliferation and induces cell apoptosis of human T24 and 5637 bladder cancer cells in vitro and in vivo," Clinical and Translational Oncology, vol. 23, no. 3, pp. 601-611, 2021.
[50] S. D. Liu, L. P. Zhong, J. He, and Y. X. Zhao, "Targeting neuropilin-1 interactions is a promising anti-tumor strategy," Chinese Medical Journal, vol. 134, no. 5, pp. 508-517, 2020.

[51] S. Roy, A. K. Bag, R. K. Singh, J. E. Talmadge, S. K. Batra, and K. Datta, "Multifaceted role of neuropilins in the immune system: potential targets for immunotherapy," Frontiers in Immunology, vol. 8, p. 1228, 2017.

[52] C. Li, P. Jiang, S. Wei, X. Xu, and J. Wang, "Regulatory T cells in tumor microenvironment: new mechanisms, potential therapeutic strategies and future prospects," Molecular Cancer, vol. 19, no. 1, p. 116, 2020.

[53] L. Cantuti-Castelvetri, R. Ojha, L. D. Pedro et al., "Neuropilin1 facilitates SARS-CoV-2 cell entry and infectivity," Science, vol. 370, no. 6518, pp. 856-860, 2020.

[54] J. L. Daly, B. Simonetti, K. Klein et al., "Neuropilin-1 is a host factor for SARS-CoV-2 infection," Science, vol. 370, no. 6518, pp. 861-865, 2020.

[55] R. Fu, W. Du, Z. Ding et al., "HIF- $1 \alpha$ promoted vasculogenic mimicry formation in lung adenocarcinoma through NRP1 upregulation in the hypoxic tumor microenvironment," Cell Death \& Disease, vol. 12, no. 4, p. 394, 2021.

[56] H. Guo, P. German, S. Bai et al., "The PI3K/AKT pathway and renal cell carcinoma," Journal of Genetics and Genomics, vol. 42, no. 7, pp. 343-353, 2015.

[57] E. W. Joseph, C. A. Pratilas, P. I. Poulikakos et al., "The RAF inhibitor PLX4032 inhibits ERK signaling and tumor cell proliferation in a V600E BRAF-selective manner," Proceedings of the National Academy of Sciences, vol. 107, no. 33, pp. 14903-14908, 2010.

[58] Z. Xia, M. Dickens, J. Raingeaud, R. J. Davis, and M. E. Greenberg, "Opposing effects of ERK and JNK-p38 MAP kinases on apoptosis," Science, vol. 270, no. 5240, pp. 1326-1331, 1995.

[59] A. J. Galliher and W. P. Schiemann, "Src phosphorylates Tyr284 in TGF- $\beta$ type II receptor and regulates TGF- $\beta$ stimulation of p38 MAPK during breast cancer cell proliferation and invasion," Cancer Research, vol. 67, no. 8, pp. 3752-3758, 2007.

[60] A.-K. Olsson, A. Dimberg, J. Kreuger, and L. Claesson-Welsh, "VEGF receptor signalling? in control of vascular function," Nature Reviews Molecular Cell Biology, vol. 7, no. 5, pp. 359-371, 2006.

[61] C. Hu and X. Jiang, "Role of NRP-1 in VEGF-VEGFR2-independent tumorigenesis," Targeted Oncology, vol. 11, no. 4, pp. 501-505, 2016.

[62] S. Rizzolio, N. Rabinowicz, E. Rainero et al., "Neuropilin-1dependent regulation of EGF-receptor signaling," Cancer Research, vol. 72, no. 22, pp. 5801-5811, 2012. 